\title{
Factores de riesgo de enfermedad cardiovascular en una población de escolares chilenos: I parte: lípidos séricos en 552 niños y adolescentes de 6-15 años*
}

Q.F. Cristián Milos G. ${ }^{1}$; M.C. Víctor Casanueva E. ${ }^{2}$; Q.F. Raúl Campos G. ${ }^{1}$; B.Q. Ximena Cid C. ${ }^{3}$; B.Q. M. Sc. Víctor Silva $\Gamma^{2}{ }^{1} ;$ M.C. Whaldo Rodr iguez Y.2; M.C. María Soledad Rodr íguez C. ${ }^{2}$

\author{
Cardiovascular disease risk factors in school children at Concepción, Chile: \\ Parte one: serum lipids in 552 children and adolescents \\ between 6 and 15 years old
}

\begin{abstract}
The distribution of serum cholesterol (TC), low density lipoprotein (LDL) cholesterol, high density lipoprotein (HDL)-cholesterol and triglycerides (TG), and the prevalence of risk-considered values were studied in school children from Concepción. Chile (6 to 15 years old; 326 boys, 226 giris). The mean TC levels were $146 \mathrm{mg}$ d d for boys and $154 \mathrm{mg} / \mathrm{dl}$ for girls but $9.1 \%$ of the sampled children had TC values between 175 and $190 \mathrm{mg} / \mathrm{dl}$, and $10 \%$ were over $190 \mathrm{mg} / \mathrm{dl}$. Mean LDL-C was $83 \mathrm{mg} / \mathrm{dl}$ in boys and $89 \mathrm{mg} / \mathrm{dl}$ in girls; $6 \%$ of samples showed values between 130 and $159 \mathrm{mg} / \mathrm{dl}$, and $1.3 \%$ over $160 \mathrm{mg} / \mathrm{dl}$. Mean HDL-C was $46 \mathrm{mg} / \mathrm{dl}$ for boys and $48 \mathrm{mg} / \mathrm{dl}$ for girls. The mean TG values were $80 \mathrm{mg} / \mathrm{dl}$ for boys and $93 \mathrm{mg} / \mathrm{dl}$ for giris.

(Key words: cardiovascular risk factors, serum lipid, childhood, total cholesterol, LDL-cholesterol, HDL-cholesterol, triglycerides.)
\end{abstract}

Las enfermedades cardiovasculares (ECV) constituyen la primera causa de mortalidad general en Chile ${ }^{1}$. En la etapa preclínica de la enfermedad coronaria (EC) se han encontrado numerosos factores de riesgo que tienen valor pronóstico. A partir de los estudios epidemiológicos realizados en Framingham ${ }^{2}$, el riesgo de desarrollar EC se ha asociado a factores como: hipercolesterolemia, hipertensión arterial, obesidad y otros. De estos factores, en los trabajos del "Coronary Heart Disease-Lipid Research Clinics Program" (LRCP) se ha establecido que existe una clara relación entre hipercolesterolemia $y$ la incidencia de $\mathrm{EC}^{3}$. La cardiopatia arteriosclerótica comienza a manifestarse, generalmente, alrededor de la cuarta

1. Departamento de Bioquímica Aplicada. Facultad de Farmacia, Universidad de Concepción.

2. Departamento de Pediatría, Facultad de Medicina, Universidad de Concepción.

3. Laboratono Central, Hospital Guillerno Grant Benavente, Concepción.

* Proyecto financiado por la Dirección de Investigación de la Universidad de Concepción (Proyecto 22.72.02) y por la Firma Boehringer Marnheim. década de la vida ${ }^{4}$; sin embargo, se ha demostrado que los factores de riesgo están presentes desde temprana edad ${ }^{5}$, habiéndose comproba. do, incluso, la presencia de lesiones ateromatosas en las coronarias de los niños ${ }^{6}$.

En Chile no existen estudios similares comparativos; por lo tanto, es necesario conocer cuál es la incidencia de los factores de riesgo de ECV en nuestra población de escolares básicos y su importancia en pediatría. El propósito de esta comunicación es presentar, en una primera etapa, la distribución de los valores séricos de colesterol total (CT), colesterol de lipoproteínas de baja densidad (LDL-C), colesterol de lipoproteinas de al ta densidad (HDLC) y triglicéridos (TG) en una población escolar de 6 a 15 años, investigación basada en el protocolo de la Organización Mundial de la Salud (OMS), para los proyectos de estudio de precursores de aterosclerosis?

\section{MATERIAL Y METODO}

Entre los años 1986 al 1988 se estudió una población de aproximadamente 1.000 escolares de ambos sexos, aparentemente sanos, de 3 escuelas públicas de la ciudad de Concepción (Chile), que proceđían 
de estratos socioeconómicos medio y bajo. Los alumnos fueron examinados por pediatras docentes y médicos residentes del Departamento de Pediatría de la Facultad de Medicina de la Universidad de Concepción. Previamente se obtuvo la autorización de la Secretatía Regional Ministerial de Salud, de la Secretaria Regio. nal Ministerial de Educación, de la Dirección del Servicio de Salud Concepción-Arauco, de la dirección de cada escuela y de los padres de los escolares, después de una entrevista y charla para explicarles los objetivos y proyecciones del prograna.

Se confeccionó una ficha clínica individual con los datos de las anamnesis, examen físico, estado nutricional y datos de laboratorio. El peso se midió en el escolar descalzo; las niñas en blusa y falda y los varones en pantalón. La talla se midjó según las normas del Ministerio de Salud y la presión arterial, según normas preestablecidas ${ }^{8}$ con esfigmomanómetros de metcurio y con el escolar en posición sentada. A los escolares, previo ayuno de doce horas, se les extrajo sangre para determinar lípidos y lipoprote ínas séricas en el Departamento de Bioquímica Aplicada de la Facultad de Farmacia de la Universidad de Concepción; los sueros fueron separados por centrifugación a velocidad baja, mantenidos a $4^{\circ} \mathrm{C}$ y analizados antes de tres dias.

E] colesterol y los TG fueron determinados por métodos enzimáticos (CHOD-PAP Boehringer Mannheim, en presencia de EDTA disódico $8 \mathrm{mM}$, y GOP-PAP, respectivamente). Para separar la fracción $\mathrm{HDL}$, tas lipoproteínas que contienen APO-B fueron precipitadas de $1 \mathrm{ml}$ de suero con $40 \mu \mathrm{l}$ de heparinato sódico de $5.000 \mathrm{U} / \mathrm{ml}$ (sigma grado 1 ) y $100 \mu \mathrm{lde} \mathrm{MnCl}_{2} 1 \mathrm{M}^{9}$. La mezcla permaneció 30 min a $4^{\circ} \mathrm{C}$ y se centrifugó a $12.000 \mathrm{rpm}$ por 2 min (microcentr ífuga Eppendorf).
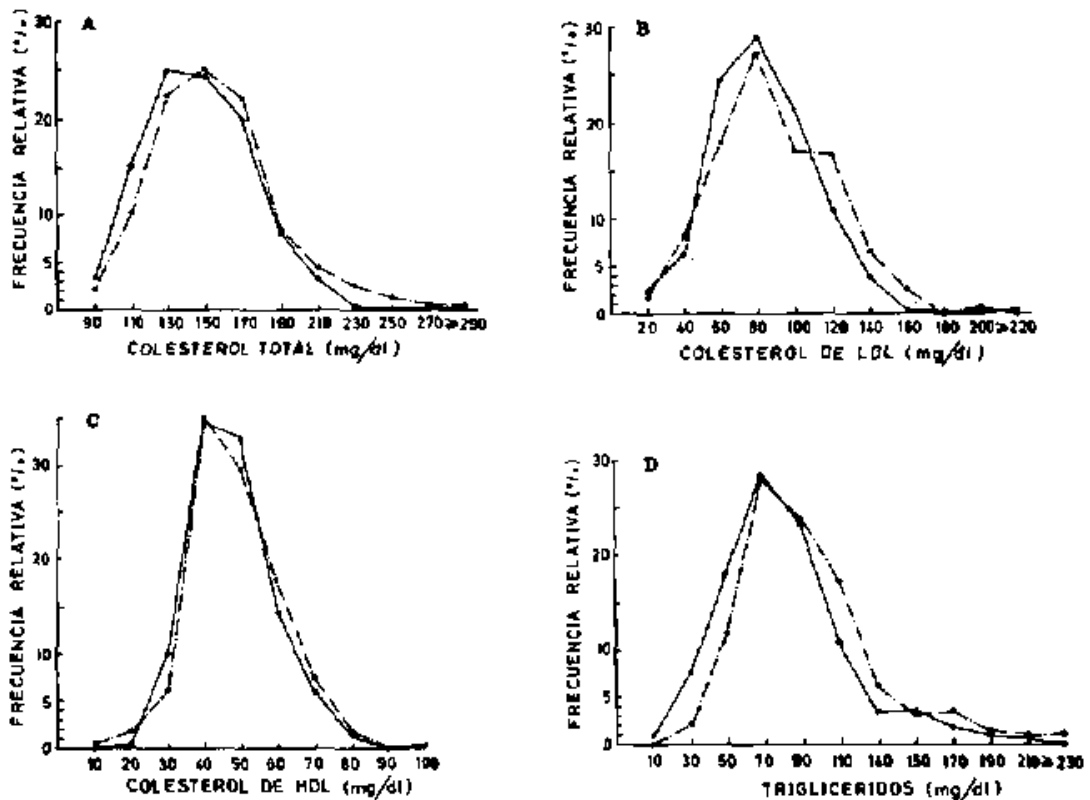

Figura 1: Distribución de las concentraciones de lípidos séricos en 552 escolares, de 6 a 15 años de edad en Concepción, Chile. A: Colesterol total: B: Colesterol de LDL; C: Colesterol de HDL; D: Triglicéridos. Niños (*-一), $\mathrm{n}=326$. Niñas $(\longrightarrow), \mathrm{n}=226$. 
Tabla 1

Promedios y desviación estándar de las concentraciones de lípidos ( $\mathrm{mg} / \mathrm{dl}$ ) en $\mathbf{5 5 2}$ escolares de 6-15 años de Concepción-Chile

\begin{tabular}{|c|c|c|c|c|c|c|c|c|}
\hline \multirow[b]{2}{*}{$\begin{array}{l}\text { Promedio } \\
\text { (mg/dl) }\end{array}$} & \multicolumn{2}{|c|}{$\begin{array}{l}\text { Colesterol } \\
\text { total }\end{array}$} & \multicolumn{2}{|c|}{ LDL-colesterol } & \multicolumn{2}{|c|}{ HDL-colesterol } & \multicolumn{2}{|c|}{ Triglicéridos } \\
\hline & $\begin{array}{l}M \\
146 *\end{array}$ & $\begin{array}{l}F \\
154\end{array}$ & $\begin{array}{l}M \\
83^{* *}\end{array}$ & $\begin{array}{l}F \\
89 * *\end{array}$ & $\begin{array}{l}M \\
46\end{array}$ & $\begin{array}{l}F \\
48\end{array}$ & $\underset{* \neq * 80}{M}$ & $\begin{array}{l}F \\
93 * * *\end{array}$ \\
\hline $\begin{array}{l}\text { Desviación } \\
\text { estándar }\end{array}$ & 29,9 & 33,4 & 29,1 & 32,3 & 10,7 & 12,2 & 34,8 & 39,6 \\
\hline
\end{tabular}

$\mathbf{M}=$ Masculino, $n=326$

$\mathrm{F}=$ Femenino, $\mathbf{n}=226$

* $\quad{ }_{\mathrm{p}}<0,005$

$* *=\mathrm{p}<0,05$

$* * *=p<0,001$

mujeres, respectivamente, siendo las diferencias estadísticamente significativas ( $p<0,005)$. Los percentiles se presentan en la tabla 2 . Los percentiles 90 y 95 , empleados habitualmente en adultos como valores de referencia para detectar la hipercolesterolemia ${ }^{13}$, fueron 184 y $198 \mathrm{mg} / \mathrm{dl}$ en hombres y 198 y $220 \mathrm{mg} / \mathrm{dl}$ en mujeres.

Si se consideran los valores entre los percentiles 2,5 y 97,5 ( $95 \%$ de la población), como rango de referencia tendríamos valores entre 95 y $205 \mathrm{mg} / \mathrm{dl}$ en hombres y 99 y $223 \mathrm{mg} / \mathrm{dl}$ en mujeres. Los valores mínimo y máximo fueron 84 y $305 \mathrm{mg} / \mathrm{dl}$ en hombres y 93 y $284 \mathrm{mg} / \mathrm{dl}$ en mujeres.

La distribución de los niveles de LDL-C se muestra en la figura 1B. Los promedios y des. viación estándar se presentan en la tabla 1 y los percentiles, en la tabla 2 . Los promedios fueron $83 \pm 29,1$ y $89 \pm 32,3 \mathrm{mg} / \mathrm{dl}$ para hombres $y$ mujeres, respectivamente, siendo las diferencias estadísticamente significativas ( $p<0,05$ ). Los percentiles 95 fueron $130 \mathrm{mg} / \mathrm{dl}$ en hombres $y$ $143 \mathrm{mg} / \mathrm{d}$ en mujeres. Las nirinas, lo mismo que ocurrió con el colesterol total, presentaron un desplazamiento hacia los valores altos que se hace evidente desde el percentil 75 (10-16 mg/dl mayor que los niños); en cambio, hasta el percentil 50 no hay diferencia significativa. La distribución de los valores en los nif̂́os fue cercana a la normal, siendo la mediana $2 \mathrm{mg} / \mathrm{dl}$ menor que el promedio; en cambio, en las niñas fue 5 $\mathrm{mg} / \mathrm{dl}$ menor. El 95\% de los niños presentó valo.

Tabla 2

Percentiles de las concentraciones de lipidos séricos (mg/dl) en 552 escolares de 6.15 años de Concepción-Chile

\begin{tabular}{|c|c|c|c|c|c|c|c|c|c|c|c|c|c|c|}
\hline \multirow{2}{*}{$\begin{array}{l}\text { Parámetros } \\
\text { CT }\end{array}$} & \multirow{2}{*}{$\frac{M^{P 2}, 5}{95}$} & \multirow{2}{*}{$\frac{5 \mathbf{F}}{99}$} & \multirow{2}{*}{$\frac{M^{p 5}}{103}$} & \multirow{2}{*}{$\frac{F}{108}$} & \multirow{2}{*}{$\frac{M^{P 10}}{110}$} & \multirow{2}{*}{$\frac{F}{116}$} & \multirow{2}{*}{$\frac{M^{P 50}}{145}$} & \multirow{2}{*}{$\frac{F}{151}$} & \multirow{2}{*}{$\frac{M^{P 75}}{166}$} & \multirow{2}{*}{$\frac{F}{174}$} & \multirow{2}{*}{$\frac{M^{P 90} F}{184198}$} & \multirow{2}{*}{$\frac{M^{P 95} F}{198220}$} & \multicolumn{2}{|c|}{$\mathbf{M}^{\mathrm{P97,5}} \mathbf{F}$} \\
\hline & & & & & & & & & & & & & 205 & 223 \\
\hline $\mathrm{LDL}-\mathrm{C}$ & 29 & 30 & 40 & 39 & 50 & 49 & 81 & 84 & 102 & 112 & 119131 & $130 \quad 143$ & 139 & 155 \\
\hline $\mathrm{HDL}-\mathrm{C}$ & 28 & 26 & 31 & 30 & 34 & 35 & 45 & 46 & 53 & 55 & $62 \quad 64$ & 6670 & 69 & 74 \\
\hline TG & 29 & 40 & 33 & 47 & 43 & 55 & 75 & 86 & 86 & 107 & 122142 & $150 \quad 175$ & 173 & 193 \\
\hline $\begin{array}{l}\text { M } \\
\text { CT } \\
\text { LDL-C } \\
\text { HDL-C } \\
\text { TG }\end{array}$ & $\begin{array}{l}\text { Mascul } \\
\text { Coleste } \\
\text { Coleste } \\
\text { Coleste } \\
\text { Triglice }\end{array}$ & $\begin{array}{l}\text { lino: } \\
\text { erol t } \\
\text { erol } \\
\text { erol }\end{array}$ & $\begin{array}{l}=32 \\
\text { al. } \\
\text { lipop } \\
\text { lipop }\end{array}$ & roteí & $\mathrm{det}$ & ja & $\begin{array}{l}\text { dad. } \\
\text { dad. }\end{array}$ & & $\begin{array}{l}\mathbf{F} \\
\mathbf{P}\end{array}$ & & $\begin{array}{l}\text { : Femenin } \\
\text { : Percentil }\end{array}$ & $\mathrm{n}=226$ & & \\
\hline
\end{tabular}


res entre 29 y $139 \mathrm{mg} / \mathrm{dl}$ y las niñas entre 30 y $155 \mathrm{mg} / \mathrm{dl}$. Los valores mínimo y máximo fueron 15 y $265 \mathrm{mg} / \mathrm{dl}$ en hombres y 14 y $202 \mathrm{mg} / \mathrm{dl}$ en mujeres.

La distribución de los niveles de HDL.C se muestra en la figura 1C. Los promedios y desviación estándar se presentan en la tabla 1 y los percentiles, en la tabla 2. Los promedios fueron $46 \pm 10,7$ y $48 \pm 12,2 \mathrm{mg} / \mathrm{dl}$ en niños y niñas, respectivamente, con una distribución cercana a la normal y muy similar en ambos sexos, observándose diferencia sólo a nivel del percentil 97,5. El 95\% de los valores se encontró entre 28 y 69 y entre 26 y $74 \mathrm{mg} / \mathrm{dl}$ en hombres y mujeres, respectivamente. Los valores minimo y máximo fueron 24 y $82 \mathrm{mg} / \mathrm{dl}$ en hombres y 11 y $96 \mathrm{mg} / \mathrm{dl}$ en mujeres.

Se observó una buena correlación entre los valores de CT y LDL-C (figura $2 \mathrm{~A}$ ), con un coeficiente de correlación ( $r$ ) de 0,828 ; entre CT y HDL-C y entre LDL-C y HDL-C no hubo correlación.

La distribución de los valores de TG se nuestra en la figura 1D. Los promedios y destiación
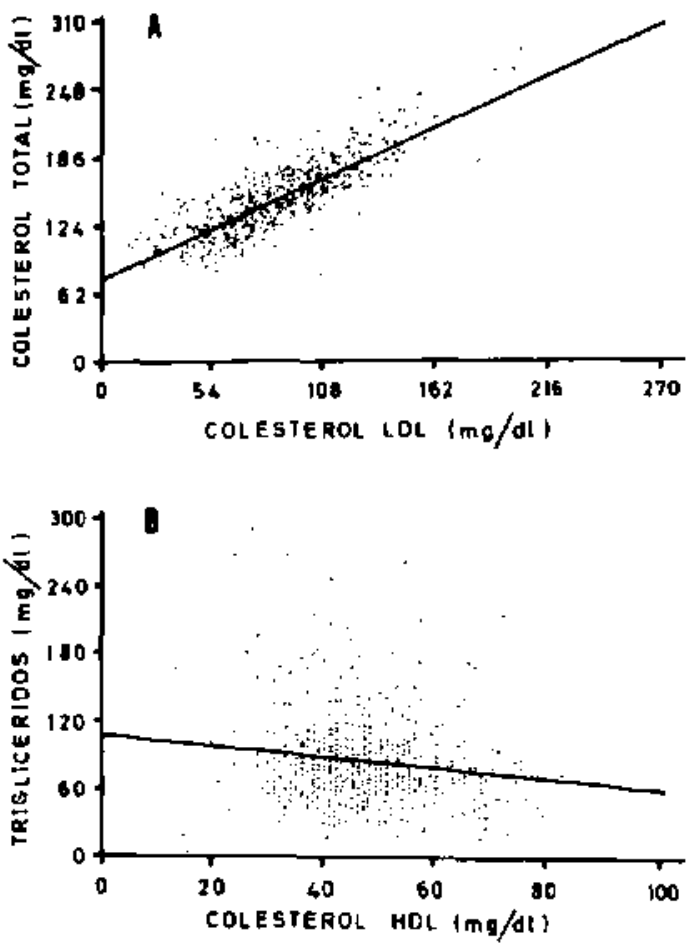

Figura 2: Correlación entre los valores de colesterol total y colesterol de LDL $(\tau=0.828)$ y entre triglicé ridos y colesterol de HDL $(r=-0,147)$ en 552 escolares de 6 a 15 años. estándar se presentan en la tabla 1 y los percentiles, en la tabla 2. Los promedios fueron $80 \pm$ 34,8 y $93 \pm 39,6 \mathrm{mg} / \mathrm{dl}$ en niños y niñas, respectivamente, siendo las diferencias estadisticamente significativas $(p<0,001)$. La tendencia hacia yalores más altos en las niñas fue más evidente aún que para el CT y LDL-C.

Se observó una correlación con tendencia negativa $(r=-0,147)$ entre los TG y HDL-C (figura 2B).

\section{DISCUSION}

Los promedios de colesterol sérico encontrados en este estudio son menores que los descritos en otros estudios poblacionales. Así, el promedio encontrado en Muscatine ${ }^{13}$ en niños y adolescentes de 6 a 18 años (ambos sexos) fue $182 \mathrm{mg} / \mathrm{dl}$; independiente de la edad, en Bogalusa ${ }^{14}$ fue de $162 \mathrm{mg} / \mathrm{dl}$ en nifos y niñas, $y$ en el estudio del LRCP fue de $162 \mathrm{mg} / \mathrm{dl}$ en niños y $165 \mathrm{mg} / \mathrm{dl}$ en niñas de 6 a 15 años $^{15}$, todos ellos realizados en Estados Unidos. Los promedios más altos de colesterol total son los informados por Vikari y $\mathrm{col}^{16}$ en niños finlandeses de 3 a 18 años: 184,1 y $189 \mathrm{mg} / \mathrm{dl}$ en niños y nif̉as, respectivamente. Más cercano a nosotros, Muñoz y col. en Venezuela ${ }^{17}$ en niños de 6 a 15 anos encontraron promedios de $153,1 \mathrm{mg} / \mathrm{d}$ l para varones y $153,6 \mathrm{mg} / \mathrm{dl}$ para mujeres, siendo los valores estables para todo rango de edad. En todos los trabajos publicados, las mujeres presentan promedios de colesterol mayores que los hombres, comportamiento que también se observó en nuestrá población, haciéndose más evidente en el percentil 95 , donde la diferencia es de $22 \mathrm{mg} / \mathrm{dl}$ en comparación con $8 \mathrm{mg} / \mathrm{dl}$ en el promedio (tablas 1 y 2 ).

Resulta difícil comparar estos trabajos, pues las técnicas empleadas son diferentes; con excepción del trabajo de Vikari ya citado, los demás utilizaron métodos colorimétricos. En el estudio de Muscatine ${ }^{13}$ las muestras de sangre fueron obtenidas sin ayuno previo. Existen otras variables individuales que dependen de la dieta y del factor socioeconómico; también existen variaciones estacionales que son debidas, en parte, a modificaciones de la dieta ${ }^{18}$. Finalmente se debe considerar la diferencia entre las determinaciones en suero y plasma ${ }^{19}$, puesto que el colesterol sérico es igual al colesterol plasmático por 1,03.

En 1987 el NCEP (National Cholesterol Education Program) publicó los acuerdos de la 
"Reunjón de expertos en detección, evaluación $y$ tratamiento de los niveles altos de colesterol en adultos"19. Para niños y adolescentes de 2 a 19 años los expertos recomendaron considerar, sin hacer diferencias de sexo, valores de colesterol de 175 a $190 \mathrm{mg} / \mathrm{dl}$ (corregidos para suero) como riesgo moderado; cifras sobre $190 \mathrm{mg} / \mathrm{dl}$ fueron consideradas como de alto riesgo ${ }^{20}$.

En relación a los TG, se acepta que su relación con la ECV es controvertida ${ }^{19,21}$, indicando que más que contribuir al riesgo de EC serian un marcador para un estilo de vida o desórdenes lipoproteicos de carácter metabólico o genético. En lo que a nuestros valores se refiere, debemos destacar que, a diferencia de los otros parámetros estudiados, nuestra población presenta valores de TG mayores que los encontrados en otros estudios poblacionales. Así, los promedios de TG publicados en el Lipid Research Clinic Data Book ${ }^{22}$ para hombres entre 5 y 9 años fueron $56 \mathrm{mg} / \mathrm{dl}$ y de 10 a 14 años, $66 \mathrm{mg} / \mathrm{dl}$, para mujeres de las mismas edades fueron de 60 y $75 \mathrm{mg} / \mathrm{dl}$, respectivamente. En Finlandia Vikari y col..$^{16}$ en nifios $y$ adolescentes de 3 a 18 años encontraron promedios menores: $28,6 \mathrm{mg} / \mathrm{dl}$ para los varones y $30 \mathrm{mg} / \mathrm{dl}$ para las mujeres.

Respecto a las lipoproteínas, Fredrickson publicó valores promedios de LDLC de 103 mg/dl para ambos sexos, desde el nacimiento has ta los 19 años $^{23}$. El estudio del LRCP ${ }^{22}$ informó para varones cifras de LDL.C de $93 \mathrm{mg} / \mathrm{dl}$ ( 5 a 9 aftos de edad) y $97 \mathrm{mg} / \mathrm{dl}$ (10 a 14 años); para mujeres, los promedios fueron $100 \mathrm{mg} / \mathrm{dl}$ (5 a 9 años) y 97 mg/dl (10 a 14 años). En Finlandia Vikari y col. ${ }^{16}$ informaron cifras $d e 116 \mathrm{mg} / \mathrm{d}$ para hombres de 3 a 18 años y de $121,5 \mathrm{mg} / \mathrm{dl}$ para mujeres de la misma edad.

Puesto que las LDL son consideradas como las lipoproteinas aterogénicas por excelen$\mathrm{cia}^{2,3,19,24,25}$ es importante analizar nuestros resultados según la opinión de los expertos del NCEP ${ }^{19,20}$, quienes recomendaron que se consideren como concentraciones deseables de LDL-C valores menores de $130 \mathrm{mg} / \mathrm{dl}$, de riesgo moderado entre 130 y $159 \mathrm{mg} / \mathrm{dl} \mathrm{y}$ de alto riesgo $>$ $160 \mathrm{mg} / \mathrm{dl}$. De acuerdo a este criterio un $6 \%$ de nuestra población escolar total presenta valores de LDL-C en la zorla considerada de riesgo moderado y un $1,3 \%$ en la de alto riesgo. Aun cuando la reunión de expertos del NCEP recomendó no considerar diferencias de edad, nj de sexo, es necesario destacar que la desviación hacia los valores altos en las niñas (figura 1B, tablas 1 y 2 ) se traduce en un porcentaje significativamente mayor con riesgo moderado y alto: 8,4 y 1,8 en comparación con 4,3 y $1,0 \%$ en niños.

Respecto a las HDL, los estudios epidemiológicos han establecido que existe correlación negativa entre su concentración sérica y la incidencia de $\mathrm{ECV}^{19,24,26,2 \mathrm{~T}}$. Nuestros valores promedios de HDL-C, 46 y $48 \mathrm{mg} / \mathrm{dl}$ en niños y niñas, respectivamente, fueron menores que los encontrados en estudios poblacionales realizados en niffos de USA ${ }^{12,22}$ y Finlandia ${ }^{16}$; es llamativo que en adultos chilenos, segín lo informado por Arteaga y col. ${ }^{28}$, esta diferencia fue aún más notoria. Morrison ${ }^{29}$ en 927 niños de 6 a 17 años obtuvo promedios de $\$ 2 \mathrm{mg} / \mathrm{dl}$ para varones y $51 \mathrm{mg} / \mathrm{dl}$ para mujeres. Fredrickson publicó cifras de HDL-C de 51 y $54 \mathrm{mg} / \mathrm{dl}$ para hombres y mujeres de 0 a 19 anlos, respectivamente ${ }^{23}$. En Finlandja ${ }^{16}$, se encontraron valores de HDL-C de $53,4 \mathrm{mg} / \mathrm{dl}$ para varones entre 3 y 18 años y de $53,8 \mathrm{mg} / \mathrm{dl}$ para mujeres de la misma edad.

Aun cuando a la fecha no se pueden sacar conclusiones, puesto que los métodos para estimar lipoproteinas no están bien estandarizados ${ }^{24}$, la "Reunión de expertos en la detección, evaluación y tratamiento de los niveles altos de colesterol sanguíneo en adultos" del $\mathrm{NCEP}^{19,24}$ otorgó prioridad al tratamiento de las concentraciones elevadas de LDL-C y recomendó que las concentraciones de HDL.C sean consideradas al comenzar a modificarse las concentraciones de LDL-C.

En la prevención de los niveles aumentados de colesterol, la altemativa más sencilla consiste en enseñar simples cambios en la composición de la dieta ${ }^{30}$. Al respecto, la Academia Americana de Pediatría recomienda una dieta variada, con mantención del peso ideal. La ingesta óptima de grasas no está determinada, pero se aconseja que constituy a el 30 a $40 \%$ de las calorias totales para asegurar el crecimiento y el desarrollo; además, se deben practicar ejercicios en forma regular y evitar el tabaquismo en los adolescentes ${ }^{31}$.

Recientemente se ha demostrado que en una población infantil estudiada diez affos más tarde, los valores de colesterol obtenidos en la niñez sirven para predecir los valores de CT y de LDLC en el adulto ${ }^{32}$. Se ha comprobado, también, que a menos de la mitad de los niños que consultan al pediatra en USA se les solicita determinación de colesterol plasmático y sólo el $35 \%$ de los adultos recuerda habérsele efectuado dicho examen ${ }^{33}$. 
Aunque nuestros promedios de colesterol son más bajos que los publicados a la fecha, es sorprendente comprobar que, de acuerdo a las recomendaciones del NCEP, el 9,1\% de los escolares estudiados presentaría moderado riesgo y el $10 \%$, alto riesgo; es decir, considerando sólo el colesterol como factor de riesgo, aproximadamente el 10\% de nuestra población se encontraría en franca situación de riesgo de ECV.

Estos resultados deberian contribuir a despertar la conciencia de los pediatras y de los médicos que atienden nifos y adolescentes acerca de la importancia de detectar los factores de riesgo de ECV en la niñez, sin dejar de considerar también el importante rol a cumplir por los otros profesionales del área de la salud y por los educadores en un plan de prevención de ECV.

\section{RESUMEN}

Se estudió la distribución de las concentraciones sêricas de colesterol total (CT), 1.DL-C, HDL-C y TG en una muestra de escol.res de Concepción, Chile (6 a 15 aftos; 326 hombres, 226 mujeres) y la prevalencia de valores considerados de riesgo. Los promedios de CT fueron 146 $\mathrm{mg} / \mathrm{dl}$ en niños y $154 \mathrm{mg} / \mathrm{dl}$ en niñas; $9,1 \%$ de la población estudiada presentó valores entre $175 \mathrm{y}$ $190 \mathrm{mg} / \mathrm{dl} \mathrm{y} 10 \%$, sobre $190 \mathrm{mg} / \mathrm{dl}$. Los promedios de L.DL-C fueron 83 y $89 \mathrm{mg} / \mathrm{dl}$, respectivamente; 6\% de la población presentó valores entre 130 y $159 \mathrm{mg} / \mathrm{dl}$ y $1,3 \%$, valores sobre $160 \mathrm{mg} / \mathrm{d} 1$. Los promedios de HDL.C fueron $46 \mathrm{mg} / \mathrm{dl}$ para niños y $48 \mathrm{mg} /$ dl para niñas. Los promedios de triglicéridos fueton $80 \mathrm{mg} / \mathrm{dl}$ en niños y $93 \mathrm{mg} / \mathrm{dl}$ en niñas. Se enfatiza en la importancia de las alteraciones lipídicas, precozmente encontradas y su implicancia a largo plazo.

(Palabras claves: lipidos séricos.)

\section{AGRADECIMIENTOS}

Este trabajo contó con la colaboración técnica del Sr. Q.F. Juan Carlos Etcheberry B., durante su permanencia en el Departamento de Bioquínica Aplicada de 1a Universidad de Concepción y al Sr. Tomás Fell B. (egresađo de Bioquímica), en el procesamiento computacional de los datos. Además, a las siguientes instituciones: Secretaría Regional Ministerial de Salud, Secretar ia Regional Ministerial de Educación, Servicio de Salud de Concepción-Asauco. Nuestro reconocimiento a la Dirección y a los padres de las Escuelas D-518, D-519, D-559 y E-1196, cuya colaboración y faciljdades nos permitieron la puesta en marcha y ejecución de este trabajo.

\section{REFERENCIAS}

1. Insfituto Nacional de Estadisticas Chile. Demografía 1984.

2. Kannel W.B., Castelli W.P., Gordon T., McNamara P.M: Serum Cholesterol, Lipoproteins and the Risk of Coronary Heart Disease. The Framingham Study. Ann Int Med 1971; 74: 1-12.

3. Heiss G., Tamir I., Davis C.E. et al: LipoproteinCholesterol Distributions in Selected North American Populations: The Lipid Research Clinics Program Prevalence Study. Circulation 1980; $61: 302-315$.

4. Solberg L.A., Strong J.P.: Risk Factors and atherosclerotic lesions: a review of autopsy. Arteriosclerosis $1983 ; 3: 187-198$.

5. Berenson G.S., Frank G.C., Hunter S.M. Srinivasan S.R., Voors A.W., Webber L.S.: Cardiovascular risk factors in children. Should they concern the Pediatrician? Am J Dis Child 1982; 136: 855-862.

6. Besenson G.S. Srinivasan S.R., Freedmon D.S. Rodhakrishnamurthy B., Dalferes E.R.: Review: Atherosclerosis and its evolution in childhood. Ametican I Med Sci 1987; 294: 429-440.

7. OMS Proyecto en Colaboración. Estudio sobre los precursores de la aterosclerosis crónica de la OMS 1980; $34: 206 \cdot 208$.

8. National Heart, Lung and Blood Institutes Task Force on Blood Pressure control in children. Report of Task Force on Blood Pressure control. Pediattics 1977; 59: 797-820.

9. Aibers J.J., Warnick G.R., Wiebe D. er al.: MultiLaboratory comparison of three heparit- $\mathrm{Mn}^{2+}$ precipitation procedures of estitnating cholesterol in high density lipoprotein. Clin Chem 1978; 24: 853-856.

10. Steele B.W., Koeflen D.F, Azar M.M., Bloszkowki T.P., Kubz $X$, Dempsey M.E.: Enzymatic determinations of cholesterol in high density 1ipoprotein fractions prepared by a precipitation technique. Clin Chem 1976; 22: 98.101.

11. Narto H.X.: The need of aceurate total cholesterol measurement. Clin Lab Med 1989;9; 37-60.

12. Rifkind B.M. Segal P.: Lipid research Clinics program reference values for hyperlipidemia and hipolipidemia. JAMA 1983: 250: 1869-1872.

13. Lauer R.M. Connor W.E., Leaverton P.E., Reiter M. Clarke W.R.: Coronary Heart Disease risk factors in school children: The Muscatine Study. J Pedjatr 1875; 86: 697-706.

14. Srinilasan S.R., Frerichs R.R., Berenson G.S.: Serum lipids and lipoprotein profile in school children from a rural community. Clin Chem Acta 1975; 60: 293-302.

15. Christensen B., Glueck C.J., Kwiterovich P.O. et od.: Plasma cholesterol and trigly ceride Distributions in 13665 Chaldren and adolescents: The Prevalence Study of the Lipid Research Clinics Program. Pediatr Res 1980: 14: 194-202.

16. Vikari J., Akerblon H.K., Nikkan T., Seppänen A. et ol.: Atheroscletosis Precursors in Finnish Children and adolescents. Act Paed Scand (Suppl) 1985; 318 : 103-109. 
17. Muñoz S., Muñoz H., Zambrano F. Gueron N.: Serum Cholesterol and Triglyceride Levels in School Age Population. Arch lnt Med 1981; 141: 24-29.

18. Buxtor J.C, Baudet M.F., Mortin C, Richard L,U., Jacotot B.: Seasonal Variations of Serum lipids and Apoproteins. Алл Nutr Metab 1988; 32: 68-74,

19. The expert Panel.: Report of the National cholesterol education program expert panel on detection, evaluation, and treatment of high blood cholesterol in adults. Arch Int Med 1988; 148: 36-39.

20. Rosenfeld L.. Atherosclerosis and the Cholesterol Connection: Evolution of a Clinical Application. Clin Chem 1989; 35 : 521-531.

21. Lippel K., Tyroler H.A., Eden H.: Meeting Summary; relationship of hypertriglyceridemia to atheroselerosis. Arteriosclerosis 1981; 1: 407-417.

22. Lipid Research Clinics Population Studies Data Book, vol 1. The Prevalence study. US Dept of Health and Human Services, National Institutes of Health Publication No 80-1527. Government Printing Office, July 1980.

23. Fredrickson D.S., Levy R.J.: Familiar hyperlipaproteinemia, in Stanbury J.B., Wyngaarden J.B., Fredrickson D.S. (eds.): The metabolic basis of Inherited Disease. New York, McGraw-Hill, 1972, p. 545 .

24. Grundy S.M., Goodman D.S., Rifkind B.M., Cleeman J.J.: The Place of HDL in Cholesterol Management. A Perspective from the National Cholesterol Education Program. Arch Int Med 1989; 149: 505-510.
25. Goldstein J.L., Ho Y.K., Basan S.K. et al.: Binding sites on macrophages that mediates uptake and degradation of acetylated low density lipoprotein, producing massive cholesterol deposition. Proc Natl Acad Sci USA 1979; 76 : 333-377.

26. Miller N.E., Forde O.H., Thelle D.S., Mfos O.D.: High density lipoprotein and coronary heart disease: a prospective case-control study. Lancet $1977 ; 1: 965-968$.

27. Gordon $T$, Costell W.P, Hortland M.C. Karmet W.B., Dewber T.R.: High density lipoptotein as a protective against coronary heart disease: The Framingham Study. An J Med 1977; 62: 707-714.

28. Arteaga A., Máz A., Donoso V., Aravena M., Acosta A.M.: El colesterol de las lipoproteínas de alta densidad del suero en una población de hombres asintonáticos, en Santiago de Chile. Rev Med Chile 1983; 111: 115.123.

29. Morrison J.A., De Groot I., Edwards B.K. et al.: Lipids and Lipoprotein in 927 School children, Ages 6 to 17 Years. Pediatrics 1978; 62: 990-995.

30. Berwick D., Creatin S., Keeler E.: Cholesterol, Children and Heart Disease: An Analysis of Alternatives. Pediatrics 1981; 68: 721-730.

31. American Academy of Pediotrics Committees on Nutrition: Prudent Lifestyle for Children Dietary Fat and Cholesterol. Pediattics 1986; 78: 521-524.

32. Laver R.M., Lee J., Clarke W.R.: Factors affecting the relationship between Childhood and Adult Cholesterol levels. The Muscatine Study. Pediatrics 1988; 82: 309-318.

33. Martin M.J., Hulley S.B., Browner W.S, Kuller L.

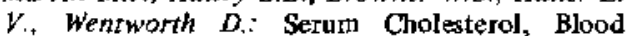
Pressure and Mortality. Implications from a Cohort of 361662 Men. Lancet 1986; 11: 933-936. 